\title{
Elderly humans exposed to concentrated air pollution particles have decreased heart rate variability
}

\author{
R.B. Devlin*, A.J. Ghio*, H. Kehrl*, G. Sanders\#, W. Cascio"
}

Elderly humans exposed to concentrated air pollution particles have decreased heart rate variability. R.B. Devlin, A.J. Ghio, H. Kehrl, G. Sanders, W. Cascio. (C)ERS Journals Ltd 2003.

ABSTRACT: Air pollution particles are thought to kill $>\mathbf{5 0 0 , 0 0 0}$ people worldwide each year. The population most at risk appears to be elderly people with respiratory and cardiovascular disease. As yet, no commonly accepted mechanism has been proposed which can explain the cause of these deaths.

Heart rate variability (HRV) was assessed in healthy elderly adults between the ages of 60 and 80 who were exposed twice for $2 \mathrm{~h}$ : once to clean air and once to concentrated ambient air pollution particles (CAPS). Changes in HRV were measured immediately before, immediately following, and $24 \mathrm{~h}$ after exposure.

Elderly subjects experienced significant decreases in HRV in both time and frequency domains immediately following exposure. Some of these changes persisted for at least $24 \mathrm{~h}$. These data were compared with HRV data collected from young healthy volunteers exposed to CAPS in a previous study, in which no CAPS-induced changes in HRV were found.

These concentrated ambient air pollution particle-induced changes in heart rate variability in a controlled human exposure study extend similar findings reported in recent panel studies and suggest potential mechanisms by which particulate matter may induce adverse cardiovascular events.

Eur Respir J 2003; 21: Suppl. 40, 76s-80s.
*National Health and Environmental Effects Research Laboratory, Environmental Protection Agency, Research Triangle Park, North Carolina, USA, ${ }^{\#}$ Division of Cardiology, University of North Carolina School of Medicine, Chapel Hill, North Carolina, USA.

Correspondence: R. Devlin, National Health and Environmental Effects Research Laboratory, MD 58D, US Environmental Protection Agency, Research Triangle Park, NC 27711, USA.

Fax: 019199666271

E-mail: devlin.robert@epa.gov

Keywords: Heart rate variability

humans

particulate matter

Received and accepted: April 122002
During the past decade, many epidemiological studies have reported statistically significant positive correlations between daily concentrations of air pollution particles with a $50 \%$ cutoff aerodynamic diameter of $10 \mu \mathrm{m}$ (PM10) and increased mortality and morbidity attributable to respiratory and cardiovascular causes [1-3]. A recent analysis of $>90$ USA cities reported that a $10 \mu \mathrm{g} \cdot \mathrm{m}^{-3}$ increase in PM10 resulted in an average increase of about $0.5 \%$ in mortality [4]. The similarity of these results is remarkable because the studies occurred in many different locations that varied significantly in geographical climate, PM10 components, time of year, and amounts of gaseous co-pollutants such as ozone. The observed increases in mortality and morbidity, while statistically significant, are still small compared to risks found in epidemiological studies of occupational or other risk factors. However, because of the large fraction of the population potentially exposed to fine particles in the air, it has been estimated that 500,000 excess deaths worldwide each year may be attributable to PM10 [5].

In general, these studies report higher mortality rates attributable to respiratory and cardiovascular causes than for total nonaccidental mortality. Elderly individuals with underlying cardiac or pulmonary disease appear to be at greatest risk [6]. These studies lend plausibility to the idea that inhalation of fine air pollution particles results in cardiopulmonary events which can rapidly trigger increased mortality or morbidity in a small fraction of the population. Two recent studies which support this idea report associations between elevated particulate matter (PM) levels and serious ventricular arrhythymias [7] as well as myocardial infarctions [8]. However, the underlying pathophysiological mechanisms which link PM and cardiopulmonary mortality remain largely unknown. It has been suggested that PM may induce lung inflammation and hypoxia, which could cause cardiovascular distress. Although some studies have observed low levels of inflammation in humans exposed to ambient air particles [9], PM-induced hypoxia has not yet been observed in humans [10].

There has been a growing recognition of the importance of the autonomic nervous system in cardiovascular death [11-14] and overall mortality in the aged [15] and among those surviving a myocardial infarction [16]. Three recent panel studies have reported associations between ambient PM levels and changes in autonomic nervous system control as assessed by heart rate variability (HRV) in elderly volunteers. HRV reflects autonomic modulation of rhythmic heart rate and changes in both the time and frequency domains are measured by a number of different analytical techniques. An association was reported between particles with a $50 \%$ cut-off aerodynamic diameter of $2.5 \mu \mathrm{m}$ (PM2.5) levels and decreased $\mathrm{HRV}$ in the high frequency (HF) range $(0.15-0.40 \mathrm{~Hz})$ in a group of 26 elderly residents of a retirement centre in Baltimore who were examined during a 3 week period in the winter of 1997. This association was limited to those participants who had some evidence of pre-existing cardiovascular disease [17]. No association was found between particles and changes in lung function, heart rate, systolic or diastolic blood pressure, oxygen saturation, or markers of inflammation as assessed by analysis of cells and fluid obtained by nasal lavage [18]. POPE et al. [19] performed repeated ambulatory electrocardiographic monitoring on seven subjects with varying degrees of respiratory and cardiovascular disease for a total of 29 person-days before, during and after episodes of elevated air pollution. Elevated PM10 levels were associated with increased mean heart rate, 
and decreased standard deviation of all beat-to-beat intervals (SDNN), a broad measure of autonomic tone. A third study investigated associations between PM and cardiovascular function in 21 elderly active Boston residents in the summer of 1997. Each person was observed up to 12 times during this period and negative associations were reported between PM2.5 and SDNN [20]. Since a decline in HRV has been associated with adverse cardiovascular events, it is possible that the lower variability associated with increased PM in these studies indicates increased risk for an acute cardiovascular event in elderly persons with compromised cardiovascular health status, via altered autonomic modulation of the cardiovascular system.

This study sought to extend the panel studies by exposing human volunteers to either clean air or relevant levels of ambient air pollution particles in a controlled manner. The recent development of ambient particle concentrators has made it possible to perform controlled exposures of animals and humans by inhalation of "real world" particles. The device used in this study concentrates particles between 0.1-2.5 microns, while gases and smaller particles are not concentrated [21, 22]. There also does not appear to be appreciable loss of individual particle components such as metal, sulphates, nitrates, acids, elemental and organic carbon, and general classes of organics [23]. In the Chapel Hill area of North Carolina, ambient particulate mass is driven primarily by mobile sources such as automobiles and is similar in size distribution and chemical composition to that found in many USA East coast cities, albeit at lower concentrations. Concentration of particles found in Chapel Hill air 6-7 fold allows controlled exposure of humans to similar concentrations of particles as seen in many major metropolitan areas.

In this study healthy elderly volunteers were exposed for $2 \mathrm{~h}$ to filtered air or PM2.5 concentrated from Chapel Hill air. Changes in HRV were assessed immediately before, immediately following, and $24 \mathrm{~h}$ after exposure. These changes were compared with HRV changes in young healthy volunteers exposed to concentrated ambient air pollution particles (CAPS) in a previous study.

\section{Materials and methods}

\section{Study population}

Both young and elderly volunteers responding to a newspaper advertisement were prescreened over the telephone. The elderly population was selected using the following criteria: age between 60-80 yrs; in good general health; no diagnosis of a cardiovascular or chronic lung disease. Specific exclusion criteria were: indication of diabetes, asthma, chronic obstructive pulmonary disease (COPD), use of $\beta$-adrenergic blocking drugs, decompensated heart failure or left ventricular ejection fraction $<40 \%$, angina, atrial fibrillation, history of nonsustained or sustained ventricular tachycardia, pacemaker, internal cardiac defibrillator, hypertrophic cardiomyopathy, infiltrate cardiomyopathy (e.g. sarcoidosis, amyloidosis), long QT interval, second or higher degree heart block, ventricular or atrial bigeminy or left ventricular hypertrophy, uncontrolled hypertension (blood pressure $>160 / 90 \mathrm{mmHg}$ ), myocardial infarction or coronary bypass surgery in the past 12 months, episodes of syncope within the past 12 months, dementia, dialysis treatment, need for supplemental oxygen, use of digoxin. The elderly subjects were also required to have visited their personal physician within the past 2 yrs and be willing to have their records reviewed to confirm self-reported diagnoses and use of medications.
The young healthy population was selected using the following criteria: between the ages of 18-40 yrs; nonsmokers for at least 5 yrs prior to study; no history of allergies or respiratory diseases (food allergy, hay fever, dust allergies, rhinitis, asthma, chronic bronchitis, COPD, tuberculosis, haemoptysis or recurrent pneumonia); and not presently on any medication prescribed by a physician (except birth control pills). A urine pregnancy test was performed on all female subjects and a positive result excluded the subject from further participation in this study.

Prior to participation in the study, subjects were informed of the procedures and potential risks and each signed a statement of informed consent. The protocol and consent form were approved by the University of North Carolina School of Medicine Committee on the Protection of the Rights of Human Subjects. The screening procedures for each subject included a Minnesota Multiphasic Personality Inventory, medical history, physical examination, chest radiography, and routine haematologic and biochemical tests.

\section{Exposure to concentrated ambient particles}

Particles between the sizes of $0.1-2.5 \mu \mathrm{m}$ present in the Chapel Hill air were concentrated 6-10 fold using a Harvard/ Environmental Protection Agency Ambient Fine Particle Concentrator. The principles by which this device works have been previously described [21, 22] and its use in human PM exposure studies has been described in detail [9]. The concentration of PM inside the chamber varied depending on concentrations of PM2.5 in the Chapel Hill air (which usually ranges from $5-30 \mu \mathrm{g} \cdot \mathrm{m}^{-3}$ ). Sham exposures were conducted by exposing subjects to clean air without particles. Each elderly volunteer was exposed twice: once to filtered air and once to CAPS. Exposures were for $2 \mathrm{~h}$ while the subject sat quietly. During the $2 \mathrm{~h}$ exposure, particle concentrations were monitored continuously at the inlet duct of the chamber by using a Tapered Element Oscillating Microbalance (TEOM, Series 1400a; Rupprecht \& Patashnick, Inc., Albany, NY). In an earlier study young volunteers were exposed a single time to either filtered air or CAPS. Subjects were monitored continuously using telemetry and arterial saturation by oxygen. Total exposure time was $2 \mathrm{~h}$. Subjects alternated $15 \mathrm{~min}$ of rest and $15 \mathrm{~min}$ of moderate exercise on a cycle ergometer during the exposure. Workload was adjusted so that these subjects breathed at a ventilatory rate, normalised for body surface area, of $25 \mathrm{~L} \cdot \mathrm{m}^{-2}$ per min.

\section{Particle characterisation}

Methods for physical and chemical characterisation of CAPS have been described earlier [9].

\section{Analysis of heart rate variability}

All potential subjects were screened with a 24-h ambulatory electrocardiogram (ECG) for the purpose of assessing their suitability for HRV analysis. Subjects were excluded if the ambulatory ECG showed evidence of atrial fibrillation, or frequent arrhythmia. Exclusion criteria also included concomitant use of medications with known effects on the autonomic nervous system and HRV, such as adrenergic receptor agonists or antagonists, calcium channel antagonists, and centrally acting antihypertensive agents. HRV was assessed immediately before and after exposure, and $24 \mathrm{~h}$ after exposure. Subjects reclined in a dark quiet room. In each 
case the final $10 \mathrm{~min}$ of this $25 \mathrm{~min}$ epoch was available for HRV analysis. The recordings were analysed on a Zymed holter system which performed initial QRS labelling and editing by standard Zymed software algorithms. An electrocardiographic research technician blinded to the treatment randomisation then edited the sequence of electrocardiographic complexes to ensure proper labelling of each QRS complex. A 7-min epoch was chosen for further analysis that minimised noise and interpolated beats. Premature atrial and ventricular beats were identified and labelled as abnormal and excluded, as were segments containing interfering noise. The remaining normal-to-normal (N-N) QRS intervals were then transferred to a second software program that downloaded the N-N files for HRV analysis. Excluded intervals were replaced using an interpolation method. Because of the stringent exclusion criteria related to arrhythmia and low noise levels, arrhythmias were rare in this population and few interpolated beats are included in the study. Nevertheless, the results of one subject were excluded because of numerous premature atrial beats.

Standard time- and frequency-domain measures were calculated and reported as measures of autonomic tone. Time-domain parameters included the SDNN and the percentage of N-N intervals $>50 \mathrm{~ms}$ (PNN50). Frequency domain analysis was limited to 7-min intervals as noted. The frequency spectrum is divided into low frequency (LF) power $(0.03-0.15 \mathrm{~Hz})$ and HF power $(0.15-0.40 \mathrm{~Hz})$. The HF component and PNN50 are believed to respond to changes in vagal input to the heart, while LF represents a mixture of both sympathetic and parasympathic input.

\section{Statistical analysis}

Data are expressed as mean \pm SE. Differences in HRV between air and CAPS-exposure for the elderly subjects were tested using a paired t-test. Significance was assumed at $p<0.05$. Changes in HRV between air and CAPS in the young subjects were tested using the paired t-test of independent means while those between multiple groups were compared using one-way analysis of variance. The post-hoc test employed was Scheffe's test. Two-tailed tests of significance were employed.

\section{Results}

\section{Study populations and exposure}

The elderly population consisted of 10 volunteers, seven males and three females, with an average age of $66.9 \pm 1.0 \mathrm{yrs}$. There was a substantial range in CAPS exposures reflecting the variation outside the facility, with individual exposures ranging from $21.2-80.3 \mu \mathrm{g} \cdot \mathrm{m}^{-3}$. The average for all CAPS exposures was $40.5 \pm 8.6 \mu \mathrm{g} \cdot \mathrm{m}^{-3}$ and the average concentration factor was 6.5. The average for air exposures was $1.1 \pm 0.4 \mu \mathrm{g} \cdot \mathrm{m}^{-3}$. The size distribution of exposure aerosols did not change following concentration and was approximately log-normal with the values of mass median aerodynamic diameter and geometric standard deviation similar to those reported previously [9].

\section{Changes in heart rate variability}

Figure 1a shows changes in HRV in elderly subjects immediately following a 2-h exposure to clean air or CAPS. Bars represent values after exposure divided by baseline values recorded immediately before exposure. Significant decreases
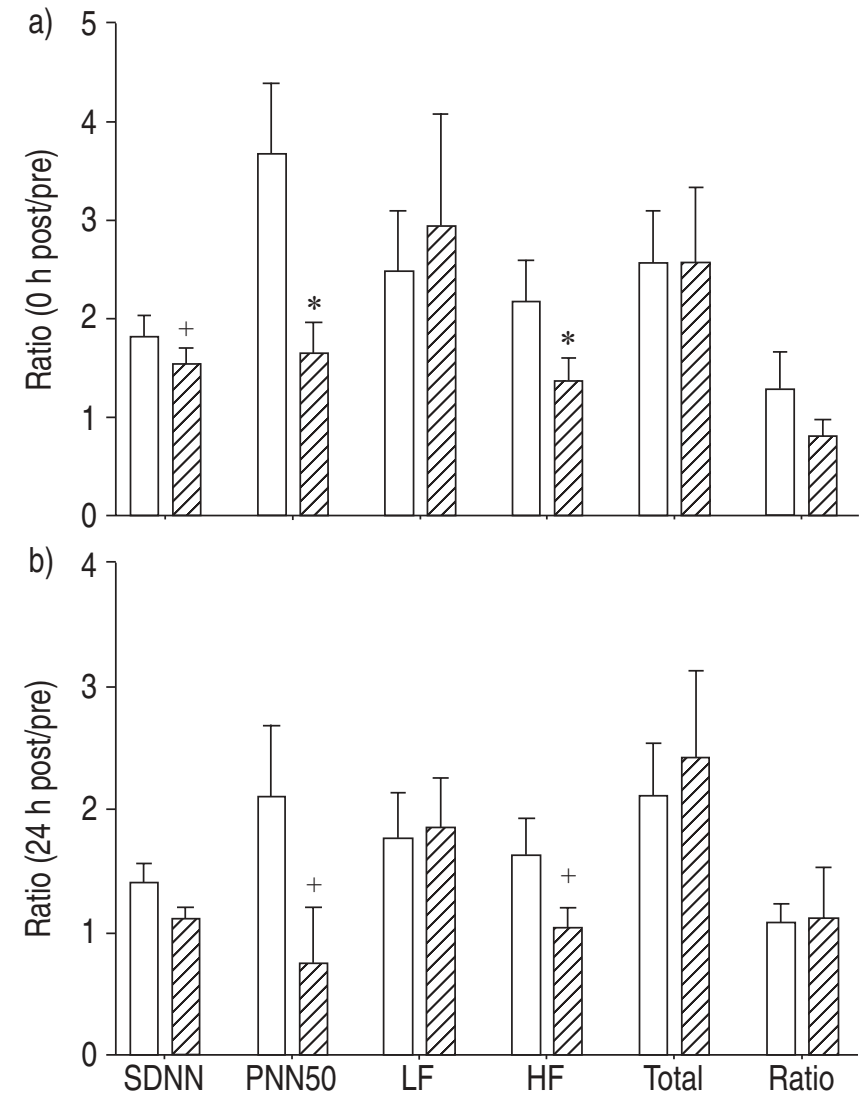

Fig. 1. - Changes in heart rate variability (HRV) in elderly volunteers a) immediately following and b) $24 \mathrm{~h}$ after exposure to concentrated ambient air pollution particles (CAPS) and clean air. Subjects were exposed to air or CAPS on two separate occasions. HRV was assessed immediately before and then a) immediately after, and b) $24 \mathrm{~h}$ after exposure. Bars labelled standard deviation of all beat-tobeat intervals (SDNN) and the percentage of N-N intervals $>50 \mathrm{~ms}$ (PNN50) refer to changes in time domain variables and bars labelled low frequency (LF), high frequency (HF), total, and ratio refer to changes in frequency domain variables. $\square$ : clean air; $\mathbb{Z}$ : CAPS. *: $\mathrm{p}<0.05 ;^{+}: \mathrm{p}<0.08$.

in both time and frequency domain were observed following exposure to CAPS, as compared with exposure to clean air. There was a $35.7 \%$ decrease in HRV in the HF domain and a 59.6\% decrease in PNN50 immediately following CAPS exposure. There was also a $16.5 \%$ decrease in SDNN immediately following CAPs exposure, which approached but did not reach statistical significance. Changes in other measurements were unchanged. CAPS-induced decreases in PNN50 were still apparent in subjects $24 \mathrm{~h}$ following exposure, and there was also a trend towards decreased HRV in the high frequency mode (fig. 1b). Abnormal beats recorded during the $24 \mathrm{~h}$ period the subjects wore the Holter monitor were also assessed. Although there were no statistically significant changes induced by CAPS for the group as a whole, several subjects experienced significant increases in abnormal beats following CAPS exposure. For example, two subjects experienced a $>3$-fold increase in premature atrial contractions following CAPS exposure, and three subjects experienced a $>5$-fold increase in bradycardia. It is not clear if these individuals represent a population that may be especially susceptible to PM or a statistical anomaly.

In an earlier study, young healthy adults were exposed to CAPS or clean air, followed by bronchoalveolar lavage [9] to assess the degree of inflammation caused by exposure to CAPS. HRV was also measured in a subset of these subjects. 
Twenty-two subjects (17 male, five female, average age $28.8 \pm 0.9$ ) were exposed to varying concentrations of CAPS (range $24.8-216.6 \mu \mathrm{g} \cdot \mathrm{m}^{-3}$ and average $105.8 \pm 12.6 \mu \mathrm{g} \cdot \mathrm{m}^{-3}$ ). Eleven subjects (seven male, four female, average age $29.6 \pm 1.1$ ) were exposed to clean air. There were no significant CAPS-induced changes in any HRV parameter in this population either immediately (fig. 2a) or $24 \mathrm{~h}$ (fig. 2b) following exposure.

\section{Discussion}

Results of this investigation demonstrate that a 2-h exposure of healthy elderly subjects to moderate levels of particulate pollution results in alterations in HRV. The levels of pollutant to which these individuals were exposed are comparable to those seen in many major metropolitan areas around the world. Furthermore, the elderly subjects were only exposed for $2 \mathrm{~h}$ while quietly sitting, in contrast to most people who would be exposed to particles for a longer period of time while ambulatory (and thus with higher respiratory rates). The physiological importance of the observed changes in HRV is not fully known. Lower HRV has been associated with increased risk for developing coronary heart disease and to sudden cardiac death. Although there is a rich literature demonstrating a link between decreased HRV and high risk
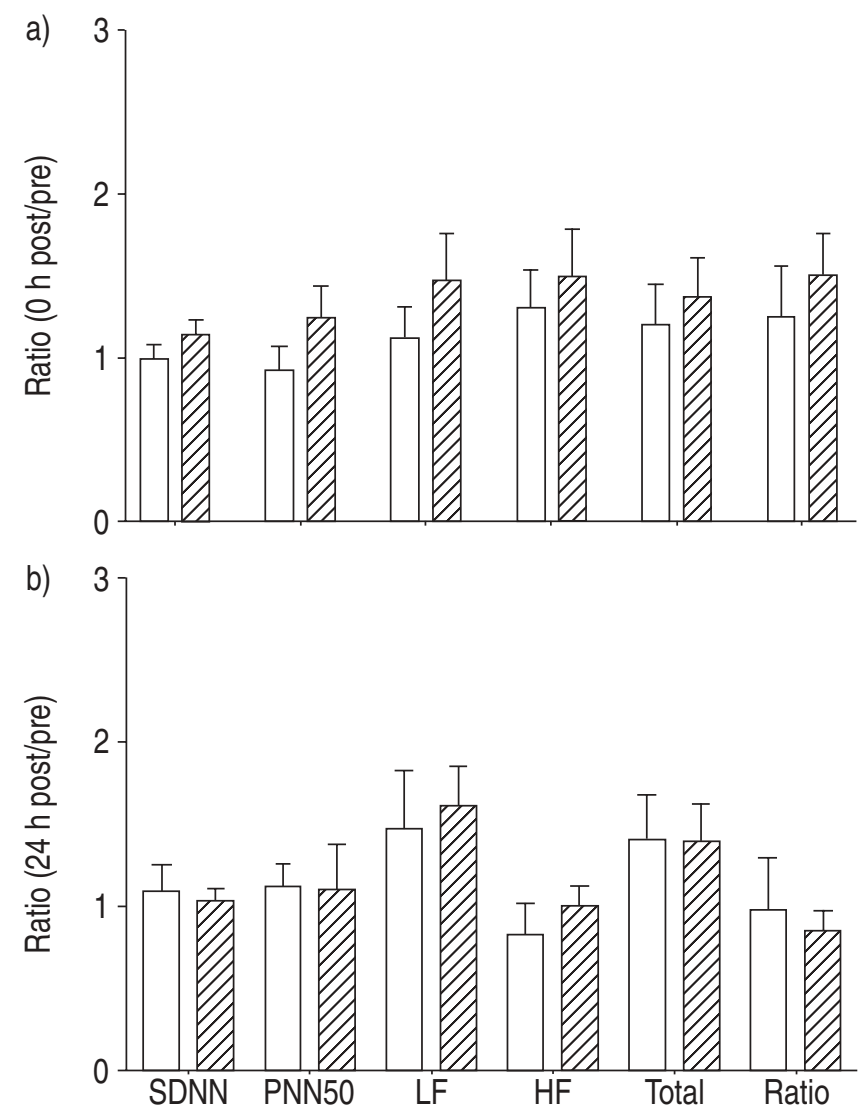

Fig. 2.-Changes in heart rate variability (HRV) in young volunteers a) immediately following and b) $24 \mathrm{~h}$ after exposure to concentrated ambient air pollution particles (CAPS) and clean air. One group of subjects was exposed to air and one to CAPS. HRV was assessed immediately before and then a) immediately after, and b) $24 \mathrm{~h}$ after exposure. Bars labelled standard deviation of all beat-to-beat intervals (SDNN) and the percentage of N-N intervals $>50 \mathrm{~ms}$ (PNN50) refer to changes in time domain variables and bars labelled low frequency (LF), high frequency (HF), total, and ratio refer to changes in frequency domain variables. $\square$ : clean air; $\mathbb{Z}$ : CAPS. of cardiovascular disease and sudden death in populationbased studies, it is not yet known whether day-to-day changes in HRV (such as those found following an acute exposure to PM) is associated with day-to-day risk for sudden cardiac death.

The effects were observed in components that reflect changes in high frequency variation in heart rate, suggesting that exposure to PM may result in a loss of parasympathetic control of heart rate. These results are in agreement with a panel study showing associations between PM and decreased HF HRV in elderly residents of a retirement home [17] and a panel study of elderly Boston residents showing associations between PM and mean of the squared differences between adjacent normal beat-to-beat (RR) intervals (r-MSSD), a measure which correlates with HF components of HRV [20]. Other panel studies have reported associations between PM and SDNN; in this study there was also a tendency for CAPs to decrease SDNN, but these levels did not reach statistical significance. These differences may reflect differences in sampling intervals ( $5 \mathrm{~min}$ in the present study versus $24 \mathrm{~h}$ in some of the panel studies), differences in the nature of the particles to which subjects were exposed (for example, CAPS do not contain significant amounts of ultrafine particles), or the fact that subjects were exposed only a single time in the present study.

These results are consistent with several recent toxicological studies which report adverse cardiac events in humans or animals exposed to PM. Changes in ECG patterns, including elevations of the ST segment, were reported in dogs exposed to CAPS [24]. Similarly altered patterns in ECG patterns were observed in rats exposed to residual oil fly ash [25] or CAPS [26]. Increased arrhythmia was also seen in rats exposed to oil fly ash or CAPS [27]. Human volunteers exposed to ultrafine carbon particles experience altered cardiac repolarisation as indicated by changes in the corrected QT interval on the ECG [28].

The concentrated ambient air pollution particle-induced decrements in heart rate variability found in healthy elderly volunteers are in contrast to the lack of response the present authors observed in healthy young adults exposed to concentrated ambient air pollution particles in an earlier study. Although both groups were exposed to comparable levels of concentrated ambient air pollution particles, the young volunteers exercised intermittently throughout the exposure, while the elderly subjects remained at rest for the entire exposure. The current authors estimate that the increased ventilation of the young subjects resulted in a $\sim 3$ fold increased deposition of concentrated ambient air pollution particles into their lungs. Nevertheless, there were no concentrated ambient air pollution particle-induced changes in heart rate variability in these subjects either immediately following exposure or $24 \mathrm{~h}$ later. This could indicate that elderly people are more responsive to particulate matter than young individuals. However, the young subject study, which was designed to primarily measure changes in lung inflammation, was not a paired study with each person serving as their own control, but rather a cohort study in which some subjects were exposed to CAPS and others to clean air. Each elderly subject was exposed to both air and concentrated ambient air pollution particles. Thus the difference in results seen between the two studies could also be related to design differences.

Acknowledgements. The authors would like to thank J. Stonehuerner and J. Scott for technical support, and M.A. Bassett, D. Levin and S. Derrenbacher for nursing support. Disclaimer: this report has been reviewed by the National Health and Environmental Effects Research Laboratory, United States Environmental Protection Agency 
and approved for publication. Approval does not signify that the contents necessarily reflect the views and policies of the Agency nor does mention of trade names or commercial products constitute endorsement or recommendation for use.

\section{References}

1. Dockery DW, Pope CA. Acute respiratory effects of particulate air pollution. Annul Rev Public Health 1994; 15: $107-132$.

2. Schwartz J. What are people dying of on high air pollution days? Environ Res 1994; 64: 26-35.

3. U.S. Environmental Protection Agency. Air Quality Criteria for Particulate Matter. 1996 EPA/600/P-95/001cf. Washington, DC, U.S. Environmental Protection Agency, 1996.

4. Samet JM, Zeger SL, Dominici F, et al. The national morbidity, mortality, and air pollution study. Part II: morbidity and mortality from air pollution in the United States. Research Report 94. Cambridge, MA, Health Effects Institute, 2000.

5. U.N. Environment Program and WHO Report. Air Pollution in the world's megacities. A Report from the U.N. Environment Programme and WHO. Environment 1994; 36 : 5-37.

6. Schwartz J. Air pollution and hospital admissions for heart disease in eight U.S. counties. Epidemiology 1999; 10: 17-22.

7. Peters A, Fröhlich O, Döring A, et al. Particulate air pollution is associated with an acute phase response in men. Results for the MONICA-Augsburg Study. Eur Heart $J$ 2001; 22: 1198-1204

8. Peters A, Liu E, Verrier RL, et al. Air Pollution and incidence of cardiac arrhythmia. Epidemiology 2000; 11: 1117.

9. Ghio AF, Kim C, Devlin RB. Concentrated ambient air particles induce mild pulmonary inflammation in healthy human volunteers. Am J Respir Crit Care Med 2000; 162: 981-988.

10. Pope CA, Dockery WS, Kanner RE, Villegas GM, Schwartz J. Daily changes in oxygen saturation and pulse rate associated with particulate air pollution and barometric pressure. Res Rep Health Eff Inst 1999; 83: 1-19.

11. Task Force of the European Society of Cardiology and the North American Society of Pacing and Electrophysiology. Heart rate variability: standards of measurement, physiological interpretation, and clinical use. Circulation 1996; 93: 1043-1065.

12. Verrier RL, Muller JE, Hobson JA. Sleep, dreams, and sudden death: the case for sleep as an autonomic stress test for the heart. Cardiovascular Research 1996; 31: 181-211.

13. Schwartz PJ. The autonomic nervous system and sudden death. Eur Heart J 1998; 19: Suppl. F, F72-80.
14. Tsuji H, Larson MG, Venditti JF Jr, et al. Impact of reduced heart rate variability on risk for cardiac events: the Framingham Heart Study. Circulation 1996; 94: 28502855.

15. Tsuji H, Venditti FJ Jr, Manders ES, et al. Reduced heart rate variability and mortality risk in an elderly cohort. The Framingham Heart. Circulation 1994; 90: 878-883.

16. Kleiger RE, Miller JP, Bigger JT Jr, Moss AJ. Decreased heart rate variability and its association with increased mortality after acute myocardial infarction. Am J Cardiol 1987; 59: 256-262.

17. Liao D, Creason J, Shy C, Williams R, Watts R, Zweidinger R. Daily variation of particulate air pollution and poor cardiac autonomic control in the elderly. Environ Health Perspect 1999; 159: 365-372.

18. Creason J, Neas L, Walsh D, et al. Particulate matter and heart rate variability among elderly retirees: the Baltimore 1998 PM study. J Expo Anal Environ Epidemiol 2001; 11: 116-122.

19. Pope CA, Verrier RL, Lovett EG, et al. Heart rate variability associated with particulate air pollution. Am Heart J 1999; 138: 890-899.

20. Gold DR, Litonjua A, Schwartz J, et al. Ambient pollution and heart rate variability. Circulation 2000; 101: 1267-1278.

21. Sioutas C, Koutrakis P, Burton RM. A technique to expose animals to concentrated fine ambient aerosols. Environ Health Perspect 1995; 103: 172-177.

22. Sioutas C, Koutrakis P, Godleski JJ, Ferguson ST, Kim CS, Burton RM. Fine particle concentrators for inhalation exposure: effect of particle size and composition. J Aerosol Science 1997; 28: 1057-1071.

23. Sioutas C, Koutrakis P, Ferguson ST. Development and evaluation of a prototype ambient particle concentrator for inhalation exposure studies. Inhal Toxicol 1995; 7: 633-644.

24. Godleski JJ, Verrier RL, Koutrakis P, Catalano P. Mechanisms of morbidity and mortality from exposure to ambient air particles. Res Report Health Eff Inst 2000; 91: 5-88.

25. Kodavanti UP, Schladweiler MC, Richards JR, Costa DL. Acute lung injury from intratracheal exposure to fugitive residual oil fly ash and its constituent metals in normo- and spontaneously hypertensive rats. Inhal Toxicol 2001; 13: 3754.

26. Gordon T, Nadziejko C, Chen LC, Schlesinger R. Effects of concentrated ambient particles in rats and hamsters: an exploratory study. Res Rep Health Effects Inst 2000; 93: 534.

27. Watkinson WP, Campen MJ, Costa DL. Cardiac arrhythmia induction after exposure to residual oil fly ash particles in a rodent model of pulmonary hypertension. Toxicol Sci 1998; 41: 209-216.

28. Frampton MW. Systemic and cardiovascular effects of airway injury and inflammation: ultrafine particle exposure in humans. Environ Health Perspect 2001; 109: 529-532. 